Rapid Reviews COVID-19

\title{
Review 2: "The effect of influenza vaccination on trained immunity: impact on COVID-19"
}

\section{Samer Singh ${ }^{1}$}

${ }^{1}$ Banaras Hindu University Institute of Medical Sciences, Centre of Experimental Medicine and Surgery, Centre of Experimental Medicine and Surgery, India

Published on: Nov 29, 2020

DOI: $10.1162 / 2 \mathrm{e} 3983 f 5.1 \mathrm{fccee} 56$

License: Creative Commons Attribution 4.0 International License (CC-BY 4.0). 


\section{$\underline{\text { RR:C19 Evidence Scale rating by reviewer: }}$}

- Potentially informative. The main claims made are not strongly justified by the methods and data, but may yield some insight. The results and conclusions of the study may resemble those from the hypothetical ideal study, but there is substantial room for doubt. Decision-makers should consider this evidence only with a thorough understanding of its weaknesses, alongside other evidence and theory. Decisionmakers should not consider this actionable, unless the weaknesses are clearly understood and there is other theory and evidence to further support it.

******************************************

\section{Review:}

The research article 'The effect of influenza vaccination on trained immunity: impact on COVID-19' medRxiv; 2020. DOI: 10.1101/2020.10.14.20212498 by Debisarun et al. explores the potential effect of populations' influenza vaccination [quadrivalent inactivated influenza vaccine Vaxigrip Tetra ${ }^{\circledR}$ (Sanofi Pasteur Europe)] on its trained immunity generation/enhancement and the potential protective (crossprotection) role in reducing the COVID-19 impact. The study is well-timed. In vitro influenza training model using peripheral blood mononuclear cells (PBMCs) from healthy donors were used to assess the effect of the influenza vaccine exposure (with or without BCG and LPS re-stimulation) by measuring select 'trained immunity' correlates (TNF- $\alpha$, IL-6, IL-1RA, IFN- $\gamma$, IL-1 $\beta$ ) at different time-points. Healthcare professionals' database was analyzed for their exposure to patients, the probability of contracting, and the subsequent duration of COVID-19. The study subjects were from less vulnerable groups and none died.

\section{COMMENTS/RECOMMENDATIONS/SUGGESTIONS:}

The study does not sufficiently support an effect of influenza vaccination on trained immunity generation or beneficial effect on the incidence, course/duration, and severity of COVID-19 due to inherent issues concerning material/details, methodology, study subjects, and the design. The title is somewhat misleading. References to relevant literature should be improved.

Some major limitations of the study concerning the main conclusions drawn are indicated below: 
CONCLUSION STATEMENT 1: "We show that a quadrivalent inactivated influenza vaccine can induce trained immunity, and the plausible mechanisms through which an enhanced antiviral state is acquired after vaccination."

Limitations 1: The statement is not supported. The pertinent controls (e.g., vaccine alone, excipients, their combinations with others enhancers/stimulants) needed for reliable head-to-head comparisons in the experiments (Fig 1 \& 2). From experiments, it may be surmised that the vaccine requires BCG to show a response. If the influenza vaccine itself cannot induce trained immunity it cannot be supposed to help acquire an antiviral state or help decrease the COVID-19 infection, duration, or intensity. In case the influenza vaccine 'Vaxigrip Tetra' used for the experiments was supposed to generate a certain level of trained immunity and the antiviral state as may be desired for its use as Influenza vaccine, the re-evaluation of the potency/efficiency and efficacy of the lot would be warranted.

Limitations 2: The background LTBI or exposure of the target population (Dutch healthcare professionals or general population) to environmental Mycobacterium is quite low [1]. The trained immunity conferred from BCG vaccination in childhood would have waned ( $<5$ years) in the majority of the adult population in the absence of a rechallenge [2-4]. So, even if the tetravalent vaccine provided any enhancement to trained immunity the majority of the population would be unable to derive the benefit.

Limitations 3: Study subjects were not from the vulnerable/elderly group. There was no change in COVID-19 disease duration in the vaccinated and unvaccinated group. So the study's potential application is severely limited.

\section{Weakness in Methodology/Description:}

1) BCG preparation used to prime PBMCs not defined correctly-live/viable, dead, purified. Whether it is live cells, killed cells, or the purified antigen derived from BCG (SSI, Denmark, $5 \mu \mathrm{g} / \mathrm{ml}$ ) is not disclosed. Different BCG preparations (live/dead/purified) have differential ability to induce/enhance trained immunity $[5,6]$.

2) Suitability detail of 'the healthy donors' for PBMCs is not provided. The sample size of donors $(n=9)$ is small. The previous exposures of individuals to environmental mycobacteria or BCG, the time elapsed since last exposure, inherent heterogeneity of the population concerning trained immunity response generation [2-4,7], could limit the applicability of study outcomes to the general population. 
3) The response was not similar in different PBMCs groups exposed to different dilutions of the vaccine (Fig 3). Inclusion of all pertinent controls was required to give confidence to the conclusions. The Vaxigrip Tetra (influenza vaccine) did not generate a response alone (0 dilutions?). It required BCG in combination with LPS for the generation of TNF response and that varied among groups. The data does not support the stimulation of trained immunity by the vaccine alone.

CONCLUSIONARY STATEMENT 2: "influenza vaccination may contribute not only to reduction of influenza but also to the COVID-19-related burden on the healthcare system. While our data show that earlier influenza vaccination is safe in relation to a later SARS-CoV-2 infection, we recommend vaccination in the absence of active COVID19 , because of the theoretical possibility to induce a cytokine storm by an enhanced immune response if the vaccine is given during an active infection."

Limitations 1:: The populations being drawn for the comparison have a differential composition (heterogeneous) and not relevant to adversely affected groups [8], so, any conclusion drawn may be likely not reproducible/ applicable to the target group. In Tables 1 and 2, the ratio of female to male is different between SARS-CoV-2 negative (2.4:1) and positive groups (3.8:1), making females about twice likely to get infected as males. The mean age of the unvaccinated and vaccinated groups is 39 and 44 years, respectively. The vulnerable elderly are not sufficiently represented in the groups.

Limitations 2: Better description of the methods/results required to increase confidence in the findings. Population proportions with underlying comorbidities were different. Proportions of the COVID-19 exposed in both groups are unequal: 37\% of unvaccinated while $80 \%$ of vaccinated had direct patient contact (Table 2). Different settings of groups are likely responsible for the observed effect (Fig. 4). Whether 'comorbidities not known' implied absence of known comorbidities or the absence of data, is not clear. The implications would be different.

Limitations 3: Age does change behavior. So, though data could be interpreted as vaccination will be more effective as even more aged vaccinated are getting less infected than younger unvaccinated, the observed effect(Fig. 4) could be a result of differential risk-taking behavior of the groups (the study authors have no control over it). Age of vaccinated and unvaccinated differed by 5 years (Table 2) despite 'all employees offered the Flu vaccination' indicating a behavioral difference - those who chose to and not chose to get vaccinated for flu.

\section{REFERENCES:}


1. Global Burden of Disease Collaborative Network. Global Burden of Disease Study 2017 (GBD 2017) Results. Seattle, United States: Institute for Health Metrics and Evaluation (IHME), 2018.

2. Menzies D. What does tuberculin reactivity after bacille Calmette-Guérin vaccination tell us?. Clin Infect Dis. 2000;31 Suppl 3:S71-S74. doi:10.1086/314075

3. Menzies D. Interpretation of repeated tuberculin tests. Boosting, conversion, and reversion. Am J Respir Crit Care Med. 1999 Jan; 159(1):15-21

4. Singh S, Maurya RP, Singh RK. "Trained immunity" from Mycobacterium spp. exposure or BCG vaccination and COVID-19 outcomes. PLoS Pathog. 2020 Oct 29;16(10):e1008969. doi: 10.1371/journal.ppat.1008969.

5. J. TURNER \& H. M. DOCKRELL. Stimulation of human peripheral blood mononuclear cells with live Mycobacterium bovis BCG activates cytolytic CD8+ T cells in vitro. Immunology 1996: 87, 339-342

6. Angelidou A, Conti MG, Diray-Arce J, et al. Licensed Bacille Calmette-Guérin (BCG) formulations differ markedly in bacterial viability, RNA content and innate immune activation. Vaccine. 2020;38(9):2229-2240.

7. Behr MA, Edelstein PH, Ramakrishnan L. Is Mycobacterium tuberculosis infection life long? BMJ. 2019;367:15770.

8. Worldometer. COVID-19 Coronavirus Pandemic.

https://www.worldometers.info/coronavirus/ 